\title{
Taming conceptual wanderings: Wilson-Structuralism
}

\section{Matteo De Benedetto ${ }^{1}$}

Received: 14 December 2020 / Accepted: 18 August 2021 / Published online: 26 August 2021

(c) The Author(s) 2021

\begin{abstract}
Mark Wilson presents a highly original account of conceptual behavior that challenges many received views about concepts in analytic philosophy. Few attempts have been made to rationally reconstruct Wilson's framework of patches and facades within a precise semantic framework. I will show how a modified version of the structuralist framework offers a semantic reconstruction of scientific theories capable of modeling Wilson's ideas about conceptual behavior. Specifically, I will argue that Theory-Elements and a modified version of Theory-Nets explicate respectively Wilson's patches and facades. I will also demonstrate how several wandering phenomena described by Wilson can be adequately reconstructed within my framework.
\end{abstract}

Keywords Mark Wilson · Structuralism · Non-statement View · Conceptual Change

\section{Introduction}

Mark Wilson (Wilson 2006, 2017) presents a highly original account of conceptual behavior that challenges many received views about concepts, reference, and conceptual change in analytic philosophy. His main point of contention with traditional understandings of concepts is the overestimation of semantic finality and conceptual mastery. Wilson argues in fact that, both in everyday life and in science, we often do not fully understand or master the concepts we use. In a detailed analysis of several conceptual histories ranging from everyday language to classical mechanics, Wilson shows the many ways in which the meanings of our terms of evaluation wander outside of our control. Behind many apparently stable macroscopic predicates such as 'weight' or 'hard' lies a very complex web of localized patches of usage. Wilson conceptualizes this patchwork structure of local conceptual usages in terms of patches and facades, i.e. pluralistic and partially indeterminate meta-conceptual units that substitute traditional philosophical understandings of theories.

$\triangle \quad$ Matteo De Benedetto

matteo.debenedetto@1rz.uni-muenchen.de

1 Munich Center for Mathematical Philosophy, Ludwig-Maximilians-Universität München,

Geschwister-Scholl-Platz 1, 80539 München, Germany 
Despite the vast praise of Wilson's work (Brandom 2010; Friedman 2010; Pincock 2010; Carus 2012), few attempts have been made to give a precise semantic reconstruction of his framework. In this work, I will show how a modified version of the structuralist view of scientific theories (Sneed 1979; Stegmüller 1976; Balzer et al. 1987; Balzer and Moulines 1996) is able to rationally reconstruct Wilson's framework of patches and facades. At first sight, the choice of reconstructing Wilson's ideas within a structuralist framework may appear quite surprising. Few frameworks in philosophy of science appear as distant as the structuralist one from Wilson's work. Wilson's reconstruction of scientific theories is in fact informal, committed to a realist understanding of scientific terms, and oriented towards scientific practice. In comparison, Structuralism in philosophy of science is characterized by a more formal approach to the subject matter, it is often combined with a somewhat anti-realist understanding of theoretical terms, and it lacks an analogous heavy focus on the practice of science. Despite these differences, I will show that there are some interesting connections between the reconstruction of scientific theories offered by Wilson and the one championed by the structuralists. Moreover, we will see that the structuralist framework, when adequately modified to eliminate its hierarchical understanding of scientific theories, is able to offer a precise semantic reconstruction of Wilson's ideas.

More specifically, I will show how my modified structuralist framework, i.e. what I will call Wilson-Structuralism, offers a semantic reconstruction of scientific theories capable of modeling Wilson's account of conceptual behavior. Specifically, I will argue that Theory-Elements and Wilson-Theory-Nets explicate respectively Wilson's patches and facades, thanks to the relaxed inter-elements constraints and the weak-specialization relationship of my framework. In order to support my claim, I will demonstrate how several wandering phenomena described by Wilson can be adequately understood in a more abstract way within Wilson-Structuralism. I will also further strengthen my case by showing how one of Wilson's main case studies of the wandering behavior of scientific terms, i.e. viscous fluids forces in classical mechanics, can be adequately reconstructed within my framework.

This work has then a three-fold aim. The first aim is to offer a rational reconstruction of Wilson's framework of patches and facades, thanks to which many of the wandering phenomena described by Wilson can be given a precise semantical understanding within a formal framework. The second aim is to offer a modified version of the structuralist framework that eliminates its original hierarchical understanding of scientific theories, offering an alternative way of reconstructing scientific theories that allows the semantic indeterminacy prescribed by Wilson. The third, more general, aim of this work is to show a surprising connection between two prima facie very different ways of reconstructing scientific theories, namely Structuralism and Wilson's framework of patches and facades.

In Sect. 2, I will summarize Wilson's work on conceptual behavior and scientific terms, focusing on his framework of patches and facades. In Sect. 3, I will present Structuralism in the philosophy of science and its model-theoretic way of reconstructing scientific theories. In Sect. 4, I will present Wilson-Structuralism, i.e. my modified structuralist framework that eliminates the hierarchical aspect of the structuralist reconstruction of scientific theories. I will show how this modified Structuralism is able to explicate Wilson's patches and facades, adequately representing several wandering- 
phenomena described by Wilson. I will also present a rational reconstruction in my framework of one of Wilson's main case studies, i.e. the case of viscous fluids forces. Finally, I will draw some conclusion about what my framework achieves, sketching some possible directions for future work.

\section{Wilson's conceptual wanderings}

Wilson expressed his views on concepts in many different places throughout his career. In my exposition, I will use as my main reference Wandering Significance (Wilson 2006), building on the tools and the terminology he used there, making connections with other related works of his when needed.

The main theme of Wilson's work is that concepts are primarily adaptive localized tools for evaluating worldly activities. Analytic philosophy, according to Wilson, has mostly analyzed concepts and other 'terms of evaluations' wrongly, not understanding their intrinsically practical and contextual nature. This misunderstanding is at the heart of what Wilson (2006, pp. 139-146) calls 'the classical picture of concepts', i.e. a list of beliefs about concepts that constitutes the received view of conceptual behavior in analytic philosophy ${ }^{1}$. Fathers of analytic philosophy like Frege and Russell may have disagreed on specific epistemological views about how we acquire concepts or how they are structured, but they shared a core of semantical presuppositions about what concepts are. Amongst the many presuppositions forming the classical picture of concepts, the most important one (and dangerous one for Wilson) are the existence of fixed and stable conceptual contents, the possibility of successfully refining unclear concepts via conceptual analysis, and the fixity of truth-values of our claims involving concepts. In other words, concepts for the classical picture are stable entities, gluing together our language with the world. Mismatches between the outside world and our representations of it arise due to our failure of correctly grasping the true essence of a given concept.

Opposing this classical picture of concepts is another general set of semantical presuppositions about concepts that Wilson (2006, pp. 236-242) calls 'anti-classical thinking'. Anti-classicists thinkers such as Quine and (the later) Wittgenstein refused completely the classical picture of concepts, replacing it with a use-based conception of concepts as directives for performing actions ${ }^{2}$. In this view, the meaning of a concept cannot be grasped in isolation but only holistically in connection with other semantical and pragmatical expressions of our language.

Wilson sees the dialectic between classical and anti-classical thinking about concepts as one of the main driving forces of the birth and the development of analytic

\footnotetext{
1 What Wilson calls the 'classical picture' of concepts should not be confused with what psychologists and philosophers of mind usually call the 'classical theory' (or traditional/definitional view) of concepts, namely the view that most concepts have definitional structure (Margolis and Laurence 1999).

${ }^{2}$ It should be noted that certain parts of Quine's philosophy may appear quite entrenched in the classical picture of concepts, such as his satisfaction with Tarskian semantics (if understood in a disquotational manner). In this work I will not consider these matters of Quinean scholarship, but it may be argued that Quine was not as monolithically anti-classical as Wilson depicts him.
} 
philosophy ${ }^{3}$. Despite their opposition, Wilson stresses that both these pictures of conceptual behavior share some unhealthy philosophical attitudes. Both classical and anti-classical thinkers suffer of what Wilson (2006, p. 126) calls the "Theory T syndrome", namely the bias of forcing all kinds of conceptual phenomena to fit into a neat single philosophical theory. The Theory $\mathrm{T}$ syndrome suppresses the individuality of individual concepts and the complexity of real world phenomena due to its obsession with monistic explanations. Thus, for instance, anti-classical thinkers like Quine, who correctly criticized the classical myth of straightforward coordination between our predicates and physical attributes, overshoot dramatically in banning any direct reference whatsoever from their philosophical views, suffering from what Wilson (2006, pp. 262-273) calls the "fear of attribute naming" and being thus condemned to a "hazy holism" (Wilson 2006, pp. 280-286) for all kinds of concepts.

Wilson's remedy for the Theory T syndrome and more generally for analytic philosophy is to make a synthesis of classical and anti-classical thinking, which he (Wilson 2006, pp. 599-605) calls (following Hume) "mitigated skepticism" about concepts. Certain concepts behave more like classical predicates, others play instead more practical roles and can therefore be understood only in a broader pragmatic context. Moreover, even the same concept can play a more classical role at a certain point of its history and a more anti-classical one at a later time. A mitigated skeptic ought not only to accept inter-conceptual pluralism in the way in which different concepts refer to the world, but also the intra-conceptual semantical "seasonalities" in the use of a given concept at different times or in different contexts. In order to have an adequate philosophical theory of how our language refers to the world, then, we have to give up entirely the idea of concepts as fixed semantical cores of our predicate usages. Wilson argues that even for the case of very simple predicates such as color ones, beneath their apparent simplicity lies a very complex web of contextual usages which cannot be grouped in a single semantical entity. So that the real crime of the Theory T syndrome is to neglect the complex 'personality' of our concepts, treating them all in the same simplistic way.

Wilson's mitigated skepticism covers both ordinary language and science. A great part of his work is devoted to showing that even in physics our apparently straightforward ways of describing the world often hide quite convoluted referential architectures. In a painstakingly detailed analysis of several macroscopic predicates of classical mechanics such as 'force' or 'hardness', Wilson shows how their usages in scientific practice exhibit a complex patchwork of localized usages tailored for the specific contexts in which they operate. The complexity of how many of our scientific terms refer is not a contingent product of a human activity, it is often the only way in which we can say something meaningful about the world. The key to understand the necessity of this semantical complexity is to acknowledge that describing the macroscopic world is a very difficult affair. The number of variables that we must take into account even in a simple macroscopic scenario of classical mechanics such as, say, the trajectory of a cannon ball is so high that describing all of them accurately requires a computational power that exceeds by far our limited intellectual capacities and measurement

\footnotetext{
${ }^{3}$ Wilson traces back the roots of the classical/anti-classical picture of concepts, as well as the related birth of analytic philosophy, to debates about the foundation of classical mechanics in the nineteenth-century. For a critical survey of Wilson's historical analysis, see (Friedman 2010).
} 
abilities. This is why the practice of science requires what Wilson (2017, Ch. 2) calls physics avoidance or variable reduction, i.e. a patchwork description of heavily simplified local domains connected in a way that efficiently reduces the complexity of the computations needed to describe a phenomenon.

\subsection{Patches and facades}

Wilson's view of conceptual behavior, both in ordinary language and in science, requires a patchwork structure that allows concepts to adapt contextually to localized usages. Wilson (2006, pp. 377-390) describes this patchwork semantical structure in terms of patches and facades.

Patches constitute the basic unit of Wilson's reconstruction of conceptual behavior. A patch is a localized mini-theory about (a specific part of) the world. A patch is composed by five different types of elements: vocabulary, domain, local reasoning tools, boundaries, and translation principles. The vocabulary of a patch is made of different linguistic entities such as predicates, names, relation symbols, and some limited logical and mathematical resources. The domain of a patch is a subset of a basic domain of physical facts, to which elements of vocabulary refer. Predicates, for instance, refer to one (or more) physical attribute(s) in the domain, while names denote constant elements. Local reasoning tools contain inferences and constraints on elements of the vocabulary that are valid within the domain of the patch. The boundaries of a patch constrain the contexts to which the patch can be applied. Finally, the translation principles are rules regulating how information can be exported and imported between a given patch and other patches connected to it.

A facade is a set of patches over a given domain of physical facts. Facades play the role of scientific theories, being collections of interconnected localized parts of our languages describing different aspects of a given phenomenon. Wilson (2006, pp. 289-296) thinks of facades as atlases of specific maps, the patches, in which every map is useful for a given purpose, but no map has the foundational, privileged role that philosophers of science often assign to a certain 'constitutive' part of a theory. Every map has its own partially distorted way of representing the world and there is no neutral epistemic perspective from which one can judge a given map to be more truthful than another one. The technical difference between facades and traditional understandings of theories lies in the inherent multi-valuedness of how elements of a facade are connected. Wilson requires only partial connections between patches of a given facade, allowing a great deal of indeterminacy in the way in which localized linguistic usages work together to achieve a global description of a phenomenon. Different patches are allowed to assign different physical referents to a common predicate, forming what Wilson (2006, p. 324) calls an uneven facade, a behavior exemplified by the concept of force in classical mechanics (Wilson 2006, pp. 158-165, 175-182). Patches can also block the export of certain inferences or reasoning tools at the boundaries, so that two patches can share a common predicate that refers to the same attribute(s) in every patch, but the inferences connected with this predicate may change from one patch to the other. Wilson (2006, p. 324) calls this inference-blocking behavior a Stokes facade, since a paradigmatic example of it is the Stokes phenomenon in optics (Wil- 
son 2006, pp. 319-327). Patches can furthermore have partially or fully overlapping domains and they are allowed to give radically different descriptions of the same subset of the physical domain. Thanks to adequate translation principles, constraining the exchange of information between the patches, these different descriptions do not produce an inconsistency. This is how contemporary multi-scalar models in engineering science manage to describe the complex behavior of some materials (Wilson 2017, Ch. 1). Patches can also be connected in such a way that a given predicate figures in the vocabulary of several interconnected patches without any common inter-patch reference, i.e. referring to different attributes in every patch it figures. This phenomenon is called by Wilson (2006, p. 273) quasi-attributes or ghost properties and it is strikingly exemplified by the concept of hardness (Wilson 2006, pp. 335-355). More generally, facades must be thought as very dynamical entities, whose structure of interconnected patches changes on the basis of the practical needs of scientific research, creating new patches that often force the overall structure of a facade to adjust itself.

Wilson offers then a deflationary understanding of scientific conceptual behavior centered around the notions of facades and patches. The partial and plural connections between patches of a given facade make the overall structure dynamically revisable according to the practical needs of science, leaving space for the inevitable conceptual wanderings necessary for this very human activity.

\section{Structuralism in philosophy of science}

In the last section, I have described Wilson's view of conceptual behavior and his framework of patches and facades. As mentioned in the Introduction, I will rationally reconstruct Wilson's framework within a modified version of the structuralist framework. In this section I will briefly give an overview of Structuralism in philosophy of science, in its original form, while in the next section I will present my modified framework.

The research program of Structuralism, understood as the model-theoretical way of reconstructing scientific theories that started with the work of Sneed, has been presented in different forms throughout the years (Sneed 1979; Stegmüller 1976; Balzer et al. 1987; Balzer and Moulines 1996). In my presentation I will use as my main reference (Balzer et al. 1987), which is arguably the most mature and complete presentation of the structuralist program.

As the name suggests, the key idea of the structuralist program is that scientific theories are best reconstructed as certain kinds of structures. This structure-centered view was originally supposed to constitute a non-statement view of scientific theories, contrasting with (what allegedly was) the logical empiricists' statement view orthodoxy ${ }^{4}$. Scientific theories are best reconstructed not as a bundle of statements, but instead as a collection of set-theoretic structures.

Specifically, the smallest unit of scientific theories reconstruction in the structuralist framework is a theory-element, i.e. a ordered pair $T=\langle K, I\rangle$ where $K$

\footnotetext{
${ }^{4}$ It should be noted that recent scholarship has convincingly argued that the opposition between statement and non-statement view of scientific theories has been largely overstated by the proponents of non-statement views. For recent perspectives bridging this alleged opposition see (Schurz 2014a, b; Andreas 2014).
} 
is a theory-core and $I$ is the set of intended applications of the theory element ${ }^{5}$. Theory-elements represent law-like scientific statements and are the building blocks of the structuralist reconstruction of scientific theories. A theory-core is a quintuple $K=\left\langle M_{p}(T), M(T), M_{p p}(T), G C(T), G L(T)\right\rangle$ that represents the theoretical framework of a given theory element, including its conceptual framework, its models, its possible empirical applications, the connections between its different applications, and the relations between the theory-element and other theory-elements of the same scientific theory.

Formally, the first component of a theory core $M_{p}(T)$ is the class of potential models of the theory-element, i.e. the set of structures of the type $\left\langle D_{1}, \ldots, D_{k}, A_{1}\right.$ $\left., \ldots, A_{k}, n_{1}, \ldots, n_{p}, t_{1}, \ldots, t_{q}\right\rangle$ that satisfy the non-law-like axioms (i.e. the statements expressible via typifications and characterizations, Balzer et al. (1987), pp. 14-17) of the theory, where $D_{1}, \ldots, D_{k}$ are sets of empirical objects, $A_{1}, \ldots, A_{k}$ sets of mathematical objects, $n_{1}, \ldots, n_{p}, t_{1}, \ldots, t_{q}$ (respectively) non-theoretical and theoretical relations between members of the two kinds of sets (usually functions from empirical objects to mathematical ones) ${ }^{6}$. Potential models are thus the conceptual framework of a given theory-element, representing the kind of entities for which it is meaningful to ask whether they satisfy the law-like statement represented by the theory-element. The second component of the theory core $M(T)$ is the class of models of the theory element, i.e. the subset of potential models including all and only the ones satisfying the law-like axioms (i.e. the statements that are neither characterizations or typifications) of the theory-element. Intuitively, models are the entities for which the law-like statement represented by the theory-element is true. Then we have the class of partial potential models of the theory-element $M_{p p}(T)$, i.e. the set of structures of type $\left\langle D_{1}, \ldots, D_{k}, A_{1}, \ldots, A_{k}, n_{1}, \ldots, n_{p}\right\rangle$ for which $\left\langle D_{1}, \ldots, D_{k}, A_{1}, \ldots, A_{k}, n_{1}, \ldots, n_{p}, t_{1}, \ldots, t_{q}\right\rangle$ is a potential model of $T$. Partial potential models are thus truncated potential models, in which the only relations remaining are the non-theoretical ones. Intuitively, they represent the empirical entities to which the law-like statement of the theory-element can be applied. The global constraint of the theory-element $G C(T)=\bigcap\left\{C_{1}(T), \ldots, C_{n}(T)\right\}$ is the combination of all constraints $C_{1}, \ldots, C_{n}$ between different applications of the theory-element. A constraint $C$ is the set of admissible combinations of potential models of the theory element, i.e. $C \subseteq \wp\left(M_{p}\right)$ such that $C \neq \emptyset, \emptyset \notin C$, and $\forall x \in M_{p}:\{x\} \in C$. Intuitively, constraints represent physical and conceptual requirements between different applications of the law-like statement represented by the theory-element. Examples of constraints are the equality constraint for mass in classical particle mechanics or the extensivity of energy in simple equilibrium thermodynamics. Finally, the last component of a theory-element is its global link $G L(T)=\bigcap\left\{L_{1}(T), \ldots, L_{n}(T)\right\}$, i.e. the

\footnotetext{
5 Here I use the first of several simplifications of the structural framework that are needed to keep the presentation of this complex framework contained, namely, I identify theory-elements with what the structuralist actually call idealized theory-elements (Balzer et al. 1987, pp. 89-92). In what follows I will consistently use the idealized version of empirical claims, theory-nets, and all the other related notions.

${ }^{6}$ For simplicity, I do not present here the structuralist way of distinguishing theoretical and non-theoretical relations/predicates of a theory. The distinction is centered around the notion of a $T$-admissible method of determination (Balzer et al. 1987, pp. 47-78) and its exposition would require the introduction of several auxiliary notions. I chose thus not to inflate the presentation of the structuralist framework, assuming the possibility of making this distinction.
} 
intersection of all abstract and concrete links $L_{1}(T), \ldots, L_{n}(T)$ between (components of potential models or potential models of) the theory-element $T$ and (components of potential models or potential models of) other theory-elements $T_{1}, \ldots, T_{n}$ relevant for the theory-element. Links $L \subseteq M_{p} \times M_{p}^{\prime}$ are admissible combinations of potential models of different theory elements, i.e. constraints between different theory-elements ${ }^{7}$. Intuitively, they represent constraints that certain law-like scientific statements of a given theory impose on their law-like neighbors for maintaining the consistency of the overall theory, such as the kinematical axioms which determine position and the chronological conditions for the measurement of time in classical particle mechanics.

If the theory-core is the theoretical framework of a theory-element, then the set of intended applications $I$ is its empirical part. The set of intended applications represents in fact the empirical circumstances to which a given theory-element is intended to apply. Formally, the set $I$ is a subset of the class of partial potential models of the theory-element, but it is meant to be only pragmatically defined as a list of paradigmatic examples that a theory has to cover in order to be successful. The success of a given theory-element can be formally expressed by the related empirical claim of a theory-element, defined as $I \in C n(K)$ (the set of intended applications is included in the content of the theory-element), where $\operatorname{Cn}(K):=$ $r(K)(\wp(M(T)) \cap G C(T) \cap \wp(G L(T)))$ is the content of the theory-element. The content of the theory element denotes the empirical parts of the potential models satisfying all the laws, links, and constraints of a theory element. Formally, it is the set of sets of partial potential models obtained by applying the operation $r(K): M_{p} \rightarrow M_{p p}$ (i.e. the operation that prunes potential models of their theoretical terms) to the members of the set $\wp(M(T)) \cap G C(T) \cap \wp(G L(T))$ (i.e. the set of all models that satisfy constraints $G C(T)$ and all inter-theoretical links $G L(T))$.

In order to clarify all these model-theoretic definitions, I will present a simple example of a theory-element, namely the one reconstructing classical particle mechanics (C PM) (Balzer et al. 1987, pp. 103-107). This theory-element is the foundational unit of the structuralist reconstruction of classical mechanics, representing the most general presentation of Newton's second-law of motion. For brevity, I will not discuss the intertheoretical links of $C P M$, focusing only on the self-contained components of the theory-element. The paradigmatic intended applications of the classical particle mechanics theory-element are the solar system, the pendulum, the projectile, and the harmonic oscillator (all members of the set $I(C P M)$ ). A potential model of classical particle mechanics is constructed as follows: $x$ is a $M_{p}(C P M)$ iff:

- $x=\left\langle P, T, S, \mathbb{N}, \mathbb{R}, c_{1}, c_{2}, s, m, f\right\rangle$

- $P$ is a finite non-empty set, $T, S$ are non-empty set, $\mathbb{N}, \mathbb{R}$ denote respectively the set of natural and real numbers.

- $c_{1}: T \rightarrow \mathbb{R}$ and $c_{2}: S \rightarrow \mathbb{R}^{3}$ are bijective and they denote respectively the coordination function for time and space.

- $s: P \times T \rightarrow S$ denotes the position function, $c_{2} \circ s_{p} \circ \breve{c_{1}}$ is smooth for all $p \in P$.

\footnotetext{
7 Here I am again simplifying the presentation of the structuralist framework, omitting the definition of concrete links (i.e. links between single components of partial potential models) and cutting a degree of complexity in the definition of a global link. For a full presentation of intertheoretical links see (Balzer et al. 1987, pp. 57-62, 78-79)
} 
- $m: P \rightarrow \mathbb{R}^{+}$denotes the mass function and $f: P \times T \times \mathbb{N} \rightarrow \mathbb{R}^{3}$ denotes the force function (made of $\mathbb{N}$ force components).

This is then the conceptual framework of the theory-element representing Newton's second law of motion in its general form, making precise the kind of entities that may satisfy Newton's law. A model of classical particle mechanics is then a potential model that actually satisfies Newton's second law. More precisely, $x$ is a $M(C P M)$ iff:

- $x=\left\langle P, T, S, \mathbb{N}, \mathbb{R}, c_{1}, c_{2}, s, m, f\right\rangle$ is a $M_{p}(C P M)$

- $\forall p \in P$ and $a \in \mathbb{R}: m(p) D^{2} r(p, a)=\Sigma_{i \in \mathbb{N}} f\left(p, \breve{c}_{1}(a), i\right)$ (i.e. Newton's second law of motion).

An example of a constraint for $C P M$ is the equality constraint for force. This constraint requires the $i$-th component force acting on a given particle at a given time to be the same independently from the system to which the particle belongs:

$$
\begin{gathered}
C(C P M)=\left\{X \mid \emptyset \neq X \subseteq M_{p}(C P M) \wedge \forall x, y \in X, \forall p, t, i\right. \\
\left.\left(p \in P_{x} \cap P_{y} \wedge t \in T_{x} \cap T_{y} \wedge i \in \mathbb{N} \rightarrow f_{x}(p, t, i)=f_{y}(p, t, i)\right)\right\}
\end{gathered}
$$

Theory-elements are then organized in larger units of scientific theories reconstruction called theory-nets. Theory-nets are collections of theory-elements sharing a significant part of their structure, organized via a relation of specialization. Intuitively theory-nets are meant to reconstruct large scientific theories, such as classical particle mechanics tout court. These large scientific theories are thought as hierarchies of more and more specialized theory-elements, representing specific sub-fields of applications of the more general theory-element in which more restrictive conditions hold (e.g. Hooke's law for elastic forces). Formally, a theory-net is a poset (i.e. a partially ordered set) $N=\langle\bar{T}, \sigma\rangle$, where $\bar{T}$ is a non-empty, finite set of theory-elements and $\sigma: \bar{T} \times \bar{T}$ is a specialization relation such that $T^{\prime} \sigma T\left(T^{\prime}\right.$ is a specialization of $\left.T\right)$ iff $M_{p}\left(T^{\prime}\right)=M_{p}(T), M_{p p}\left(T^{\prime}\right)=M_{p p}(T), M\left(T^{\prime}\right) \subseteq M(T), G C\left(T^{\prime}\right) \subseteq G C(T)$, $G L\left(T^{\prime}\right) \subseteq G L(T)$, and $I\left(T^{\prime}\right) \subseteq I(T)$. Thus a theory-net is a collection of theoryelements sharing the same classes of potential and partial potential models and partially ordered in terms of set-theoretic inclusion of their classes of models, admissible combinations of potential models (both intra- and inter-theory element ones), and intended applications.

Structuralists stress that further restrictions should be imposed on the specialization relation of the theory-elements of a given theory-net for harmonizing the empirical claims of theory-elements into a substantial global claim sharing a common empirical ground. Two important restrictions that contribute to this harmonization of empirical claims are connectedness, i.e. $\forall T_{i}, T_{j} \in \bar{T} \exists T_{k_{1}}, \ldots, T_{k_{n}}$ such that $\left(T_{i} \sigma T_{k_{1}} \vee T_{k_{1}} \sigma T_{i}\right) \wedge$ $\ldots \wedge\left(T_{j} \sigma T_{k_{n}} \vee T_{k_{n}} \sigma T_{j}\right)$, and having a singleton-basis, i.e. $B(N)=\{T \mid T \in \bar{T} \wedge$ $\left.\forall T^{\prime} \in \bar{T}\left(T \neq T^{\prime} \rightarrow \neg T \sigma T^{\prime}\right)\right\}$ contains exactly one element. A connected theory-net having a singleton-basis is called a theory-tree. The most detailed example of scientific theory reconstruction in the structuralist program, i.e. classical particle mechanics, is a theory-tree. Theory-trees can thus be considered the paradigmatic structuralist unit of reconstruction of big scientific theories. It is important to note that the two 
harmonizing restrictions that theory-nets must satisfy in order to classify as theorytrees, i.e. connectedness and singleton-basis, strengthen the hierarchical aspect of the structuralist reconstruction of scientific theories. Scientific theories that can be reconstructed as theory-trees have in fact a single, most general and most fundamental theory-element (i.e. the singleton-basis) of which every other theory-element is a specialization. Every other law-like statement of the scientific theory so reconstructed is then understood as merely specifying more restrictive conditions under which the fundamental law holds.

As in the case of theory-elements, I will present a simple example of a theory-net, namely a (very small) part of the theory-tree reconstructing the whole of classical mechanics. The CPM theory-element that we have seen before is the singleton-basis of the classical particle mechanics theory-tree and thus all the other theory-elements are specializations of $C P M$, imposing further constraints on $C P M$-models, constraints, and links. The very detailed structuralist reconstruction of the $C P M$ theory-tree (Balzer et al. 1987, pp. 180-191) singles out four different main lines of specializations of the CPM theory-element, namely symmetry forces, position-dependent forces, velocity-dependent forces, and time-dependent forces. In what follows, I will briefly present only two theory-elements belonging to the velocity-dependent forces part of the theory-tree, for both their simplicity and their relevance for Wilson's case studies. A simple example of a specialization of $C P M$ is given by the theory-element of velocity-dependent classical particle mechanics ( VCPM). The models of $V C P M$ are models of $C P M$ in which at least one component force of a given particle depends on the particle velocity. Formally, $x$ is a $M(V C P M)$ iff:

- $x=\left\langle P, T, S, \mathbb{N}, \mathbb{R}, c_{1}, c_{2}, s, m, f\right\rangle$ is a $M(C P M)$;

- $\exists p \in P, i \in \mathbb{N}$ such that for all $a \in \mathbb{R}: f\left(p, \breve{c}_{1}(a), i\right)=F(\operatorname{Dr}(p, a), a)$;

- $\exists p, a$ and $j \in\{1,2,3\}$ such that $D_{j} F(\operatorname{Dr}(p, a), a) \neq 0$.

Another specialization of $C P M$, which is also a specialization of $V C P M$, is the theory-element of simple frictional classical particle mechanics ( $F C P M)$. The models of SFCPM are models of VCPM (and thus of $C P M$ ) in which at least one component of frictional force is determined by a power of the velocity alone. Formally, $x$ is a $M(S F C P M)$ iff:

- $x=\left\langle P, T, S, \mathbb{N}, \mathbb{R}, c_{1}, c_{2}, s, m, f\right\rangle$ is a $M(V C P M)$;

- $\exists p \in P, i \in N$ such that for all $a \in \mathbb{N}: f\left(p, \breve{c}_{1}(a), i\right)=b(p, i)(\operatorname{Dr}(p, a))^{l}$ with $b: P \times \mathbb{N} \rightarrow \mathbb{R}$ and $l \in \mathbb{N}, l \geq 1$.

These are thus the central notions of the structuralist framework for reconstructing scientific theories, centered around the concepts of theory-elements and theory-nets. These two notions denote the specific kinds of structures representing (respectively) localized and general scientific theories ${ }^{8}$. As it will be clear in the next section, these two notions, when adequately modified, correspond quite naturally to Wilson's notions of patches and facades.

\footnotetext{
${ }^{8}$ I must stress that in my presentation I focused only on a small (albeit central) part of the structuralist galaxy of units of scientific theory reconstructions. Important pragmatic and dynamic extensions of theorynets and theory-elements are for instance non-idealized theory-nets (Balzer et al. 1987, pp. 357-362), theory-evolutions (Balzer et al. 1987, pp. 216-221), and theory-holons (Balzer et al. 1987, pp. 387-407).
} 


\section{Wilson-Structuralism}

Having presented the structuralist framework in its original form, in this section I will show how by eliminating its hierarchical aspect one obtains a semantic view of scientific theories capable of reconstructing many of the wandering phenomena described by Wilson. Thus, I will not use Wilson's theory as a target phenomenon for a structuralist reconstruction of the kind through which structuralists analyzed several scientific theories. What I will do, instead, is to show how to change two central structuralist notions in order to make the structuralist framework able to reconstruct scientific theories with the kind of semantic indeterminacy prescribed by Wilson. More specifically, I will show how the structuralist notions of theory-elements and theorynets, when the latter is adequately modified, provide a formal equivalent of Wilson's notions of patches and facades.

I will first present my modified structuralist framework in its general form and then I will show how it can rationally reconstruct one of Wilson's main examples of conceptual wandering in classical mechanics, i.e. the case of viscous fluids forces.

\subsection{Wilson-theory-nets}

We have seen in the last section how the structuralist reconstruction of scientific theories is centered around two notions, theory-elements and theory-nets. If the former is meant to reconstruct law-like specific parts of a scientific theory, the latter organizes all these specific parts into a more coherent whole. The level of generality of these two structuralist notions exactly corresponds to the one of Wilson's patches and facades. Moreover, theory-elements, just like Wilson's patches, are micro-theories about a specific part of the world (i.e. the models of the theory-elements), made of a conceptual and linguistic part (i.e. their potential models) together with their own reasoning tools and connections with neighboring theories (i.e. their constraints and inter-theoretical links). Theory-nets, then, exactly like Wilson's facades, are collections of micro-theories (i.e. theory-elements) over a given macro-domain that organize the connections between these micro-theories by constraining their components (via their specialization relation).

Can we then easily reconstruct Wilson's patches as theory-elements and Wilson's facades as theory-nets? Unfortunately not. The problem with this tentative mapping is the aforementioned hierarchical aspect of the structuralist reconstruction of scientific theories. Theory-nets (and a fortiori theory-trees) organize, in fact, theory-elements into a hierarchical chain of specialization relation(s), where specific applications (in the intuitive sense of the word) of a theory are supposed to be always conceptually reducible to more general law-like statements. Applications of a scientific theory are then in the structuralist reconstruction of a theory just model-theoretic precisifications of the related more fundamental theory-element. Theory-nets are then a perfect example of the kind of semantic finality based on the received view of scientific theories that Wilson repeatedly attacks in his work. The whole bestiary of semantic wanderings presented in Wandering Significance can be seen as a list of ways in which the uses of scientific terms defy a structuralist-like hierarchical reconstruction of a scientific 
theory. Wilson, in fact, repeatedly shows how applications and non-fundamental lawlike statements of a scientific theory are not reducible to mere precisifications of a more general law, but they often expand, twist, and extend the uses of the scientific terms and the reasoning tools of the theory in unexpected ways. Despite the aforementioned similarities between the two notions, structuralist theory-nets cannot then (in their canonical form) adequately mimic Wilson's facades, due to their hierarchical organization of theory-elements.

In order to solve this problem, I will now present a modified structuralist framework, i.e. Wilson-Structuralism, in which I eliminate this hierarchical aspect of the structuralist reconstruction of scientific theories. I will keep the coarse-grained organization of Structuralism, but I will drastically change its representation of how the different law-like statements of a given scientific theory are organized. More specifically, I will change the definition of a theory-net and the related specialization relation. I will not require theory-elements of the same theory-net to be related by subset inclusion of models, constraints, and links, but only by the non-empty intersection between these components. This change will allow the modified theory-nets to enjoy the 'multivaluedness' needed to adequately represent several wanderings phenomena described by Wilson.

Formally, I will leave completely unchanged the structuralist definition of a theoryelement. Just like in classical Structuralism, in Wilson-Structuralism a theory-element $T$ is a couple $T=\langle K, I\rangle$ where $K=\left\langle M_{p}, M, M, G C, G L\right\rangle$ is a theory core and $I$ denotes the intended applications of the theory-element. All components of the theorycore are defined exactly like we saw in Sect. 3, as well as the intended applications.

Theory-elements in Wilson-Structuralism are meant to explicate Wilson's patches, i.e. self-contained micro-theories about a subset of a given domain. The components of a patch that Wilson describes can be adequately mapped to the ones of a given theory-element. The vocabulary of a patch is represented in Wilson-Structuralism by the potential models of the related theory-element. Potential models are in fact the conceptual framework of a given theory-element, representing the linguistic part of the law-like statement. The domain of a Wilson's patch is instead mapped to the models of the related theory-element. Models of a theory-element depict in fact in the structuralist framework the possible scenarios that satisfy the law-like statement, thus representing all the possible 'denotations' of the related scientific terms. The boundaries of Wilson's patches are instead mapped to the intended applications of a theory-element, i.e. to the pragmatically defined subset of the class of partial potential models that represents the empirical situations to which the theory-element should apply. The reasoning tools of a Wilson's patch are mapped to the constraints of the theory-element. This mapping is justified by noting that Wilson's patches are equipped with a variety of reasoning tools that encompasses also the kind of physical and conceptual requirements on scientific terms that are framed by the structuralists as constraints on the related theory-element. Moreover, it has been recently shown how more paradigmatic reasoning tools such as deductive inferences can be represented in the structuralist framework as constraints on the acceptable combinations of potential models of a theory-element (Andreas 2013). Finally, a patch translation-principles, i.e. the rules that norm the import and the export of information with other neighbor patches, are mapped to the intertheoretical links of 
the theory-element, a component that does the same exact job of Wilson's translationprinciples between different theory-elements.

If theory-elements adequately represent Wilson's patches, the role of facades is played in Wilson-Structuralism by an adequately modified version of theory-nets. The key difference between what I will call Wilson-theory-nets and the traditional definition that I presented in the last section is the specialization relation. We have seen that in traditional Structuralism the specialization relation of theory-nets hierarchically orders theory-elements by requiring (weak) subset inclusion of their models, constraints, links, and intended applications (and equality of potential models and partial potential models). Formally, in a traditional theory-net, a theory-element $T^{\prime}$ is a specialization of another theory-element $T$, i.e. $T^{\prime} \sigma T$, iff

$$
\begin{aligned}
& M_{p}(T)=M_{p}\left(T^{\prime}\right), M_{p p}(T)=M_{p p}\left(T^{\prime}\right), I\left(T^{\prime}\right) \subseteq I(T), \\
& M\left(T^{\prime}\right) \subseteq M(T), G C\left(T^{\prime}\right) \subseteq G C(T), G L\left(T^{\prime}\right) \subseteq G L(T)
\end{aligned}
$$

In Wilson-Structuralism, I replace this specialization-relation with a weaker version that I will call weak-specialization. Like orthodox specialization, weak-specialization requires equality of potential models and partial potential models, as well as weak subset-inclusion of intended applications, but it only requires non-empty intersection between the models, the constraints, and the links of the two theory-elements. This means that a theory-element is a weak-specialization of another one if they share the same conceptual framework and empirical ground (aka potential models and partial potential models), its range of applications is (weakly) included in the one of the other, and they have compatible models, constraints, and inter-theoretical links.

Formally, a Wilson-Theory-Net (WTN) is a poset $N^{W}=\left\langle\bar{T}^{W}, w \sigma\right\rangle$, where $\bar{T}^{W}$ is a non-empty, finite set of RW-theory-elements and $w \sigma: \bar{T}^{W} \times \bar{T}^{W}$ is a weak specialization relation such that $T^{\prime} w \sigma T$ ( $T^{\prime}$ is a weak specialization of $T$ ) iff

$$
\begin{aligned}
& M_{p}(T)=M_{p}\left(T^{\prime}\right), M_{p p}(T)=M_{p p}\left(T^{\prime}\right), I\left(T^{\prime}\right) \subseteq I(T), \\
& G C\left(T^{\prime}\right) \cap G C(T) \neq \emptyset, G L\left(T^{\prime}\right) \cap G L(T) \neq \emptyset, M\left(T^{\prime}\right) \cap M(T) \neq \emptyset .
\end{aligned}
$$

These new definitions allow in a Wilson-theory-net specializations of theoryelements to have different (although compatible) models, constraints, and intertheoretical links than the more general theory-element of which they are a specialization. Thus, the hierarchical structure of a traditional theory-net is maintained in a Wilson-theory-net only for what concerns the intended applications, while the theoretical relationships between the cores of the theory-elements are allowed far more diversity (modulo direct-neighbor compatibility).

This diversity is the key element that allows Wilson-theory-nets to adequately represent Wilson's facades. The relaxed constraints imposed on the components of the different theory-elements of a Wilson-theory-net allow several conceptual wanderings described by Wilson, properly understood as particular set-theoretic relationships between components of different theory-elements. In order to be represented in Wilson-Structuralism, Wilson's wanderings have in fact to be reconstructed as differences in the mathematical structure that (parts of) the related theory-elements denote. 
This is because of the differences in the degree of realism and externalism in the semantics of scientific terms between Wilson's and the structuralist framework. As I hinted in the Introduction, in fact, while Wilson freely talks about an external reality in which scientific terms take their reference by aligning themselves with a (set of) attribute(s), structuralist frameworks reconstruct instead scientific terms as certain kinds of functions or relations, i.e. as certain components of the potential models of a given theory-element. Specific reconstructions and views about the ontological status of theoretical terms vary within the structuralist camp, from arguably anti-realist reconstruction (e.g. Sneed 1979; Andreas 2014) to more neutralist approaches (e.g. Stegmüller 1976; Moulines 1991), but no specific version of the structuralist framework conceptualizes scientific terms in a strongly realist way like Wilson. In the structuralist framework, denotation of a descriptive term may be construed via the set-theoretic predicates in which the term occurs and the actual models that satisfy the corresponding set-theoretic predicate(s) (as well as all the constraints and links). Thus, Wilson's inter-patch changes in the alignment between certain predicates and the attributes they refer to have to be reconstructed, in a structuralist framework, in a more abstract way as certain kinds of differences in the structures denoted by the set-theoretic predicates of the respective theory-elements. As a guide to this abstract representation of the semantic indeterminacies described by Wilson, I will use the following metaphysical assumption:

Assumption: Two different attributes cannot be represented by the same class of mathematical entities ( $\approx$ contraposition of Leibniz's identity of indiscernibles). Therefore, if a certain predicate refers to two different attributes in two different contexts, this predicate cannot be represented by the same mathematical entity in both contexts.

The idea behind this quasi-Leibnizian assumption is that difference in scientific terms reference must have a correlate in the mathematical representation of that part of a scientific theory. For instance, if a given predicate refers to two different attributes in two different contexts (i.e. Wilson's uneven facades construction), then its mathematical representation has to be different as well in the two contexts. Differences in reference must have some discernible consequences in the logical reconstruction of a theory. Formally, in order to talk about a predicate and its representation in my framework, I use the set-theoretic projection function $\Pi\left(T, i_{1} \ldots i_{x}\right)=$ set of all entities appearing in places $i_{1} \ldots i_{x}$ in theory $T$ (cf. Balzer et al. (1987), p. 61, Moulines (1981), pp. 214-215). This function picks out from each tuple in $T$ the entities occupying the $i_{1} \ldots i_{x}$ places, thus having as a domain the set of all ordered tuples of $T$ and as a range the set of all entities occupying the places $i_{1} \ldots i_{x}$ in tuples of $T$. So, for instance, the function $\Pi(M(C P M), f)$ refers to the union of all the force functions $f$ appearing in the models of the $C P M$ theory-element, thus picking out from each tuple in $M(C P M)$ its force component. This projection function, together with the metaphysical assumption above, constitute the main tools for representing inter-patch changes of scientific term meaning in Wilson-Structuralism. Then, I will say that the meaning of a given scientific term is different in two directly connected patches if and only if the two theory-elements representing these patches, provided that they are 
directly connected by a weak-specialization relation, have models with sets of functions representing the given scientific terms that are incomparable with respect to the subset relation. Informally, this incomparability constrain assures us that the meaning of the scientific term under focus is truly different in the two related patches (and not just a specification or a generalization of each other).

We can now see how Wilson-theory-nets are able to explicate several of the wandering phenomena described by Wilson. For instance, uneven facades, i.e. facades in which some patches assign a different referent to a common predicate (such as the case of the force predicate in classical mechanics), are represented in WilsonStructuralism as Wilson-theory-nets in which (at least) two theory-elements, directly related by weak-specialization, have models with incomparable (with respect to the subset relation) sets of functions representing a given scientific term, i.e. $\exists T, T^{\prime} \in \bar{T}^{W}$ such that $T w \sigma T^{\prime}$ and $\Pi(M(T), t) \nsupseteq \Pi\left(M\left(T^{\prime}\right), t\right)$ and $\Pi(M(T), t) \nsubseteq \Pi\left(M\left(T^{\prime}\right), t\right)$. This incomparability condition implies that the union of all functions $t$ appearing in the models of $T$ is incomparable with respect to the subset relation to the union of all functions $t$ appearing in the models of $T^{\prime}$. Thus, some function $t$ in the models of $T$ has to be different from any function appearing in the models of $T^{\prime}$ and some function $t$ in the models of $T^{\prime}$ has to be different from any function appearing in the models of $T$. However, since the two theory-element $T$ and $T^{\prime}$ are in a weak-specialization relation, at least one function $t$ appearing in their models has to be the same, because of the non-empty intersection of the models required by the weak-specialization relation. This formal condition mirrors in an abstract way the fact that patches in an uneven facade drag a given predicate into matching attributes that are, relative to a common application, incompatible with its original meaning. Note that the incomparability of sets of functions appearing in the models of two theory-elements implies the incomparability of the models and thus it is in stark contrast with the structuralist orthodox specialization relation (that requires weak subset inclusion of the models).

Stokes facades, i.e. facades in which different patches validate different inferences involving a common predicate (such as the Stokes phenomenon in optics), are instead represented in Wilson-Structuralism as Wilson-theory-nets in which at least two theory-elements, directly related by weak-specialization, have the same models but incomparable (with respect to the subset relation) constraints, i.e. $\exists T, T^{\prime} \in \bar{T}^{W}$ such that $T w \sigma T^{\prime}, M(T)=M\left(T^{\prime}\right)$ and $G C(T) \nsupseteq G C\left(T^{\prime}\right)$ and $G C(T) \nsubseteq G C\left(T^{\prime}\right)$. This formal condition represents the fact that in a Stokes facade patches share the same meaning of their scientific terms (i.e. they refer to the same attributes) at the cost of limiting the validity of certain reasoning tools at the patch boundaries.

Wilson-theory-nets are also compatible with the existence of ghost properties, i.e. an extreme case of uneven facades in which a given term has a different alignment in every patch and there is no common attribute that the predicate denotes (such as the case of hardness). We can represent this phenomenon as a Wilson-theory-net where every two theory-elements have incomparable (with respect to the subset relation) sets of function representing a given theoretical term and in which the intersection between all the models of all the theory-element is empty, i.e. $\exists t$ such that $\forall T, T^{\prime} \in$ $\bar{T}^{W}\left(\Pi(M(T), t) \nsupseteq \Pi\left(M\left(T^{\prime}\right), t\right),\left(\Pi(M(T), t) \nsubseteq \Pi\left(M\left(T^{\prime}\right), t\right)\right)\right.$, and $\bigcap\{M(T) \mid T \in$ $\left.\bar{T}^{W}\right\}=\emptyset$. Moreover, the weak specialization relation $w \sigma$ of a Wilson-theory-net is designed to make possible loop-structures of specializations where no patch is 
more fundamental than another one (i.e. what Wilsons calls the "lousy encyclopedia phenomenon"). These cycles are allowed by weak specialization because, in contrast to the orthodox specialization relation, it is not anti-symmetric.

More generally, the weakening of the specialization relation and the consequent less homogeneous core-net (the net of all theoretical core of theory-elements in a given theory-net) of a Wilson-theory-net allow Wilson-Structuralism to represent several of the eerie internal organizations of scientific theories described by Wilson such as incompatible descriptions of the world, Escherian geometries of patches inter-connection, and horizontal and vertical multi-valuedness of patches. Wilsontheory-elements can for instance have the same intended applications but incomparable models, thereby representing Wilson's patches offering incompatible descriptions of the same part of the domain. Cycles of weak-specialization relations may occur together with incomparable (with respect to the subset relation) constraints, models, and links between connected theory-elements, creating multiple possible ways in which the fundamentality of a theory-element in a Wilson-theory-net can be assessed.

Wilson-Structuralism is then able to adequately represent several wandering phenomena described by Wilson, achieving a logical reconstruction of scientific theories free from the semantic finality of classical Structuralism. Theory-elements and Wilsontheory-nets rationally reconstruct Wilson's patches and facades in a precise formal framework, in which several wanderings described by Wilson can be understood in an abstract way as specific set-theoretic relations between components of different theoryelements. Moreover, Wilson-Structuralism can be seen also as a complementary generalization of (a part of) the structuralist framework. In fact, Wilson-theory-nets have as specific cases the traditionally defined theory-nets that we saw in Sect. 3, i.e. Wilson-theory-nets in which all the weak specialization relations are also traditional specialization relations. If none of the semantic wanderings described by Wilson occur between any of its theory-elements, in fact, a Wilson-theory-net is just a structuralist theory-net, where all the models, constraints, and links of the specialized theoryelements are subsets of the ones of the theory-element of which it is a specialization. As an extreme case of this lack of wanderings, we can also have a structuralist theorytree as a specific case of a Wilson-theory-net in which there is a single theory-element of which all other theory-elements are specializations. This specific case can mirror what Wilson calls a flat structure facade, i.e. a facade that presents no wandering between its patches and thus can be said to consist "essentially one patch, that covers its whole domain adequately" (Wilson 2006, p. 379).

This modified Structuralism joins other recent attempts of renewing classical Structuralism by simplifying and improving its behemothic framework. Andreas' "Carnapian Structuralism" (Andreas 2010, 2014) is an example of a kind of Structuralism more compatible with other contemporary philosophical views about scientific theories reconstruction. Carnapian Structuralism restyled the structuralist framework through a reader-friendly system of postulates built around the notion of a theoretical expansion of a partial potential model. Wilson-Structuralism takes a more radical departure from classical Structuralism than Carnapian Structuralism, radically weakening several semantic presupposition of the orthodox framework, but they both try to bring the structuralist way of reconstructing scientific theories closer to contemporary philosophy of science. 


\subsection{Taming conceptual wanderings: the case of viscous fluids forces}

In order to make clearer how Wilson-Structuralism represents Wilson's framework of patches and facades, I will sketch how one can reconstruct as a Wilson-Theory-Net one of Wilson's main case studies of wandering referents in science, namely viscous fluids 'forces' in classical mechanics.

I have stressed in Sect. 2 how a considerable part of (Wilson 2006) is dedicated to show how the apparently neat theory-structure of classical mechanics hides a complexity of wandering patches of usage ingeniously connected in a facade-like way. One of Wilson's (Wilson 2006, pp. 157-165, 175-182) favorite examples of this semantic phenomenon is the concept of force. Through a detailed analysis of several subfields of classical mechanics, Wilson shows that this central concept of Newtonian physics is remarkably prone to change physical referents from one application to another one. In particular, the efforts of nineteenth-century physicists in extending Newtonian mechanics to more and more macroscopic phenomena pushed the predicate 'force' to be attached to attributes radically differing from any true force. Wilson (2006, pp. 158159) stresses for instance the case of viscous "forces", in which the predicate force denotes net losses and gains of momentum caused by molecules leaving or entering the fluid "particle". It is only thanks to what Wilson (2017, p. 368) calls a computational opportunity, then, that the behavior of fluids can be described with mathematical tools analogous to the ones used in more traditional parts of classical mechanics (cf. Wilson (2006), pp. 175-176). This computational opportunity, together with the aforementioned pivotal change of reference of the force predicate, allowed then physicists to claim that the Navier-Stokes equations for viscous fluids are just a specialization of Newton second law of motion, thereby annexing the underlying behavior of viscous fluid to the phenomena adequately described by classical mechanics. Analogous strategies of theory expansion via 'property dragging' are behind the case of frictional and elastic forces (Wilson 2006, pp. 175-176), parts of classical mechanics where the predicate force gets attached respectively to a net effect cause by the strength of the substratum and to a measure of internal stress.

In Wilson's terminology, the changes of referents for the predicate force in different parts of classical mechanics are a paradigmatic case of uneven facade, in which 'force' refers to different physical attributes in the viscous fluid, the frictional, and the elastic patches of classical mechanics. Given the metaphysical assumption in the last subsection, this change of referent implies different mathematical representations of the force predicate in the logical reconstruction of these parts of classical mechanics. So that structuralist theory-elements representing these 'forces' must have incomparable sets of force functions (and thus incomparable models). This incomparability is explicitly forbidden in the classical structuralist reconstruction of classical particle mechanics, where the models of every theory element have to be a subset of the ones satisfying Newton's second law of motion (i.e. the fundamental $C P M$ theory-element). This limitation in the models makes every force function in the models of any theory-element in $C P M$ just a specialization of the force function in the foundational theory-element corresponding to Newton's second law of motion. So that structuralists are forced to either reconstruct the viscous fluids and the frictional force theory-elements within $C P M$, 
characterizing them as simple additions of further constraints on a given component of the force function (just like we saw for the velocity-dependent forces theory-element in Sect. 3) or to reconstruct these parts of classical mechanics as belonging to different, albeit related by suitable links, theory-nets. The latter option is exemplified by Moulines' (Moulines 1981, 2013) reconstruction of thermodynamics as (what he calls) a theory-frame. Both options, from Wilson's perspective, are not adequate reconstructions, since they either hide the change in meaning of the force predicate as a simple specification (the former reconstruction within the same theory-net) or they unnaturally divide the connected usages of force in classical mechanics into several theory-nets (the latter, multi-theory-nets type of reconstruction). Both kind of reconstruction are thus examples of the kind of semantic finality in traditional logical reconstructions of classical mechanics that Wilson $(1998,2014)$ argues against. The concept of force used in applying classical mechanics to viscous fluids cannot be a mere specialization of the one employed in Newton's second law of motion since the physical attribute denoted by the predicate is radically different from the one to which force aligns itself in $C P M$. At the same time, viscous fluids forces, according to Wilson, should be reconstructed together with all the other forces in classical mechanics, since they are part of the same facade obtained by gradual extensions of the force predicate in new domains (i.e. the phenomenon that Wilson calls "property-dragging nucleation", cf. Wilson (2006), p. 194).

Wilson-Structuralism allows this difference in the interpretation of the force predicate to be adequately reconstructed within a single Wilson-theory-net. We have in fact seen that Wilson-Theory-Nets can be uneven facades, i.e. Wilson-theory-nets in which (at least) two theory-elements, directly connected by a weak-specialization relation, have models with incomparable (with respect to the subset relation) sets of functions for a given term (and thus incomparable models). So that, in reconstructing classical particle mechanics in Wilson-Structuralism, the viscous fluid theory-element $V$ isc C P M can belong to the same theory-net of the C P M theory-element, the former being a weak-specialization of the latter that assigns a different meaning to the force function. More formally, since the force predicate denotes different physical attributes in the fundamental $C P M$ patch and in the viscous fluids patch, we can assume that the $C P M$ theory-element and the $\operatorname{ViscCP} M$ theory-element (respectively representing the two patches in Wilson-Structuralism) have incomparable sets of force functions in their models: $\Pi(M(C P M), f) \nsubseteq \Pi(M(V i s c C P M), f)$ and $\Pi(M(C P M), f) \nsupseteq \Pi(M(V i s c C P M), f)$. Then, the $V i s c C P M$ theory-element can still be a weak-specialization of the $C P M$ theory-element (or of another theoryelement connected to $C P M$ such as the velocity-dependent force theory-element), since in Wilson-Structuralism a theory-element can be a weak-specialization of another one despite having incomparable models (a condition implied by having incomparable sets of functions in the models). So, that, assuming that the CPM and the ViscCPM theory-element have at least one model jointly satisfying them and that viscous fluids forces can be mathematically reconstructed with the same typification of the force occurring in the CPM potential models (both conditions seem intuitively justified by the aforementioned existence of the computational opportunity described by Wilson), we can say that the viscous fluid theory-element is a weak-specialization of the classical particle mechanics theory-element: ViscC PM $w \sigma C P M$. 
This sketch of a case study shows how Wilson-Structuralism is able to reconstruct Wilson's framework of patches and facades, making precise in which sense in certain parts of classical mechanics the force predicate is attached to deviant attributes. The case study focused on the case of viscous fluids, but analogous theory-elements forming uneven facades can be built for the aforementioned cases of frictional and elastic forces. Furthermore, Wilson-Structuralism can adequately reconstruct in the same way other kinds of wandering phenomena described by Wilson. For instance, as already mentioned in the last subsection, Stokes facades, i.e. facades where different patches validate different inferences involving a common predicate, can be represented by Wilson-Theory-Nets having at least two theory-elements that have the same models but incomparable constraints. Thus, one could reconstruct Wilson's paradigmatic example of a Stokes facade, namely the Stokes phenomenon (Wilson 2006, pp. 319327), through three different theory-elements, sharing the same models but having as incomparable constraints the tree dominant behaviors of the light intensity predicate described by Wilson. Extreme uneven facades that include ghost properties, i.e. predicates with a different interpretation in every patch and no core meaning, such as the one describing the behavior of the hardness predicate (Wilson 2006, pp. 335-355) can be similarly reconstructed by Wilson-theory-nets in which for a given predicate such as 'hard' every possible pair of theory-elements have incomparable sets of functions in their models and the overall intersection of the models of all theory-elements of the Wilson-theory-net is empty.

\section{Conclusion}

Let me recap the main steps of the present work. Starting from Wilson's analysis of the complex ways in which language refers to the world, I presented his framework of patches and facades. I then pointed to the surprising connections between two central notions of the structuralist reconstruction of scientific theories, i.e. theory-elements and theory-nets, and Wilson's patches and facades. We have seen however that the heavily hierarchical aspect of the structuralist framework poses a problem for any tentative structuralist reconstruction of Wilson's notions. I then presented my modified version of the structuralist framework, i.e. Wilson-Structuralism. I showed how this modified structuralist framework, relaxing the definition of a theory-net and the related specialization relation, is able to eliminate the hierarchical aspect of original Structuralism. We have then seen how in Wilson-Structuralism theory-elements and Wilson-theorynets adequately represent Wilson's notions of patches and facades, allowing a precise reconstruction of several wandering phenomena described by Wilson such as the case of viscous fluids forces in classical mechanics.

Wilson-Structuralism achieves then a precise semantic reconstruction of many conceptual wanderings described by Wilson and a structuralist reconstruction of scientific theories more compatible with the nuances and the dynamics of scientific practice. Wilson-theory-nets provide in fact a more general alternative to the orthodox notion of theory-net, by allowing one to reconstruct within the same theory-net radical change of meanings and other conceptual wanderings that might be present in the target phenomena. Wilson-theory-nets and Wilson-Structuralism tout court should then be thought as 
a further tool in the structuralist toolbox that allows a precise semantic reconstruction of several semantic indeterminacies that can be found in many scientific theories.

In connection to these achievements, various directions for future work present themselves. A natural extension of the present work is to expand the scope of Wilson-Structuralism, taking into account also more pragmatic structuralist notions of scientific theory reconstruction such as theory-evolution, paradigm-driven theory-nets, and crystallizations (Moulines 2011, 2013, 2014). From these extensions, I expect Wilson-Structuralism to achieve interesting complementary alternatives of these structuralist notions, allowing a vast range of semantic indeterminacies within their logical reconstruction of scientific theories. For instance, I expect this expanded WilsonStructuralism to be able to model dynamic wanderings such as semantic detoxification (Wilson 2006, pp. 545-552), asymptotic connections between patches (Batterman 2001; Wilson 2017), and Machian explications (Wilson 2012a; Carus 2012). Other promising ways of extending this framework would be to add linguistic and pragmatic contexts in order to model also Wilson's context-adjusting models of predicate extension (Wilson 1982, 2012b) or to merge Wilson-Structuralism with Carnapian Structuralism (Andreas 2014, 2020) and with accounts of deductive reasoning in structuralist frameworks (Andreas 2013) in order to model Wilson's contextual notion of inference validity and logical inconsistencies (Wilson 1994, 2000). It would also be interesting to merge Wilson-Structuralism with other reconstructions of scientific theories devised to capture the indeterminacy of theoretical terms such as Carnap's $\epsilon$-term methodology (Carnap 1956; Schiemer and Gratzl 2016; Leitgeb and Carus 2020; Leitgeb MS).

Acknowledgements I am indebted to Hannes Leitgeb, Naftali Weinberger, and three anonymous referees for helpful comments on previous drafts of this paper.

Funding Open Access funding enabled and organized by Projekt DEAL. This research was funded by the LMU Munich's Institutional Strategy LMUexcellent within the framework of the German Excellence Initiative.

\section{Declarations}

Conflict of interest The author declares that he has no conflict of interest.

Open Access This article is licensed under a Creative Commons Attribution 4.0 International License, which permits use, sharing, adaptation, distribution and reproduction in any medium or format, as long as you give appropriate credit to the original author(s) and the source, provide a link to the Creative Commons licence, and indicate if changes were made. The images or other third party material in this article are included in the article's Creative Commons licence, unless indicated otherwise in a credit line to the material. If material is not included in the article's Creative Commons licence and your intended use is not permitted by statutory regulation or exceeds the permitted use, you will need to obtain permission directly from the copyright holder. To view a copy of this licence, visit http://creativecommons.org/licenses/by/4.0/.

\section{References}

Andreas, H. (2010). New account of empirical claims in structuralism. Synthese, 176, 311-332.

Andreas, H. (2013). Deductive reasoning in the structuralist approach. Studia Logica, 101, 1093-1113.

Andreas, H. (2014). Carnapian structuralism. Erkenntnis, 79, 1373-1391.

Andreas, H. (2020). Dynamic tractable reasoning: a modular approach to belief revision. Cham: Springer. 
Balzer, W., \& Moulines, C. U. (Eds.). (1996). Structuralist theory of science: focal issues. Walter De Gruyter, Berlin: New Results.

Balzer, W., Moulines, C. U., \& Sneed, J. (1987). An Architectonic for Science. The structuralist Program. D: Reidel Publishing Company, Dordrecht.

Batterman, R. (2001). The devil in the details: Asymptotic reasoning in explanation, reduction, and emergence. Oxford: Oxford University Press.

Brandom, R. (2010). Platforms, patchworks, and parking garages: Wilson's account of conceptual finestructure in wandering significance. Philos. Phenomenol. Res., 82(1), 183-201.

Carnap, R. (1956). The methodological character of theoretical concept. In H. Feigel \& M. Scriven (Eds.), Minnesota studies in the philosophy of science I (pp. 38-76). Minneapolis: University of Minnesota Press.

Carus, A. W. (2012). Engineers and drifters; The ideal of explication and its critics. In P. Wagner (Ed.), Carnap's ideal of explication and naturalism (pp. 225-239). Macmillan: Palgrave.

Friedman, M. (2010). Logic, mathematical science, and twentieth century Philosophy: Mark Wilson and the analytic tradition. Nous, 44(3), 530-544.

Leitgeb, H. (MS): "Ramsification and Semantic Indeterminacy". Manuscript.

Leitgeb, H. and Carus, A.W. (2020): "Rudolf Carnap. Supplement E: The Reconstruction of Scientific Theories". In Edward N. Zalta (Ed.), The Standford Encyclopedia of Philosophy (Fall 2020 Edition), forthcoming URL https://plato.stanford.edu/archives/fall2020/entries/carnap/reconstruct-scitheories.

Margolis, E., \& Laurence, S. (1999). Concepts: Core readings. Cambridge MA: MIT Press.

Moulines, C. U., et al. (1981). An example of a theory-frame: equilibrium thermodynamics. In J. Hintikka (Ed.), Probabilistic thinking, thermodynamics, and the interaction of the history and philosophy of science (pp. 211-238). Dordrecht: Reidel.

Moulines, C. U. (1991). Pragmatics in the structuralist view of science. In G. Schurz \& G. J. W. Dorn (Eds.), Advances in scientific philosophy (pp. 313-326). Amsterdam: Rodopi.

Moulines, C. U. (2011). Cuatro tipos de desarrollo teórico en las ciencias empiricas. Metatheoria, 1(2), 11-27.

Moulines, C. U., et al. (2013). Crystallization as a form of Scientific Semantic Change: The case of Thermodynamics. In B. O. Küppers (Ed.), Evolution of semantic systems (pp. 209-230). Berlin: Springer.

Moulines, C. U. (2014). Intertheoretical relations and the dynamics of science. Erkenntnis, 79(8), 15051519 .

Pincock, C. (2010). Exploring the boundaries of conceptual evaluation. Philos. Math., 18(3), 106-136.

Schiemer, G., \& Gratzl, N. (2016). The Epsilon-Reconstruction of theories and scientific structuralism. Erkenntnis, 81(2), 407-432.

Schurz, G. (2014). Criteria of theoreticity: Bridging statement and non-statement view. Erkenntnis, 79, 1521-1545.

Schurz, G. (2014). Philosophy of science: a unified approach. New York: Routledge.

Sneed, J. (1979). The logical structure of mathematical physics. Dordrecht: D. Reidel Publishing Company. Stegmüller, W. (1976). The Structure and Dynamics of Theories. Heidelberg: Springer.

Wilson, M. (1982). Predicate meets property. Philos. Rev., 91(4), 549-589.

Wilson, M. (1994). Can We Trust Logical Form? J. Philos., 91(10), 519-544.

Wilson, M. (1998). Classical Mechanics. In E. Craig (Ed.), Routledge Encyclopedia of Philosophy. London: Taylor \& Francis. https://doi.org/10.4324/9780415249126-Q068-1

Wilson, M. (2000). Inference and Correlational Truth. In M. Gupta \& A. Chapuis (Eds.), Circularity (pp. 57-73). Definition and Truth: Ridgeview Press.

Wilson, M. (2006). Wandering significance: An essay on conceptual behavior. Oxford: Clarendon Press.

Wilson, M. (2012a). The Perils of Polyanna. In P. Wagner (Ed.), Carnap's ideal of explication and naturalism (pp. 205-224). Macmillan: Palgrave.

Wilson, M. (2012b). Long Ago, in a Context Far Away. In M. Frappier, D. Brown, \& R. DiSalle (Eds.), Analysis and interpretation in the exact sciences, The Western Ontario series in philosophy of science vol 78 (pp. 57-73). Dordrecht: Springer.

Wilson, M. (2014). What Is "Classical Mechanics" Anyway? In R. Batterman (Ed.), The oxford handbook of philosophy of physics (pp. 43-105). Oxford: Oxford University Press.

Wilson, M. (2017). Physics avoidance: And other essays in conceptual strategy. Oxford: Oxford University Press. 
Publisher's Note Springer Nature remains neutral with regard to jurisdictional claims in published maps and institutional affiliations. 